Received 4 April; accepted 15 July 2002; doi:10.1038/nature01042.

1. Pearson, H. The regeneration gap. Nature 414, 388-390 (2001).

2. Baguñà, J., Saló, E. \& Auladell, C. Regeneration and pattern formation in planarians. III. Evidence that neoblasts are totipotent stem cells and the source of blastema cells. Development 107, 77-86 (1989).

3. Agata, K. \& Watanabe, K. Molecular and cellular aspects of planarian regeneration. Semin. Cell Dev. Biol. 10, 77-83 (1999).

4. Newmark, P. A. \& Sánchez Alvarado, A. Not your father's planarian: a classic model enters the era of functional genomics. Nature Rev. Genet. 3, 210-219 (2002).

5. Agata, K. et al. Structure of the planarian nervous system (CNS) revealed by neuronal cell markers. Zool. Sci. 15, 433-440 (1998).

6. Cebrià, F. et al. Dissecting planarian CNS regeneration by the expression of neural-specific genes. Dev Growth Differ. 44, 135-146 (2002)

7. Kato, K., Orii, H., Watanabe, K. \& Agata, K. The role of dorsoventral interaction in the onset of planarian regeneration. Development 126, 1031-1040 (1999).

8. Kobayashi, C., Nogi, T., Watanabe, K. \& Agata, K. Ectopic pharynxes arise by regional reorganization after anterior/posterior chimera in planarians. Mech. Dev. 89, 25-34 (1999)

9. Orii, H. et al. The planarian HOM/HOX homeobox genes (Plox) expressed along the anteroposterior axis. Dev. Biol. 210, 456-468 (1999).

10. Sánchez Alvarado, A. \& Newmark, P. A. Double-stranded RNA specifically disrupts gene expression during planarian regeneration. Proc. Natl Acad. Sci. USA 96, 5049-5054 (1999).

11. Shibata, N. et al. Expression of vasa(vas)-related genes in germline cells and totipotent somatic stem cells of planarians. Dev. Biol. 206, 73-87 (1999).

12. Umesono, Y., Watanabe, K. \& Agata, K. Distinct structural domains in the planarian brain defined by the expression of evolutionarily conserved homeobox genes. Dev. Genes Evol. 209, 31-39 (1999).

13. Cebrià, F. et al. The expression neural-specific genes reveals the structural and molecular complexity of the planarian central nervous system. Mech. Dev. 116, 199-204 (2002).

14. Ogawa, K. et al. Planarian FGFR homologues expressed in stem cells and cephalic ganglions. Dev. Growth Differ. 44, 191-204 (2002).

15. Wiedermann, M. \& Trueb, B. Characterization of a novel protein (FGFRL1) from human cartilage related to FGF receptors. Genomics 69, 275-279 (2000).

16. Fire, A. et al. Potent and specific genetic interference by double-stranded RNA in Caenorhabditis elegans. Nature 391, 806-811 (1998).

17. Amaya, E., Musci, T. J. \& Kirschner, M. W. Expression of a dominant negative mutant of the FGF receptor disrupts mesoderm formation in Xenopus embryos. Cell 66, 257-270 (1991).

18. Conlon, F. L. \& Smith, J. C. Interference with brachyury function inhibits convergent extension, causes apoptosis, and reveals separate requirements in the FGF and activin signalling pathways. Dev. Biol. 213, 85-100 (1999).

19. Smith, J. C., Price, B. M., Green, J. B., Weigel, D. \& Herrmann, B. G. Expression of a Xenopus homolog of Brachyury (T) is an immediate-early response to mesoderm induction. Cell 67, 79-87 (1991).

20. Isaacs, H. V., Pownall, M. E. \& Slack, J. M. eFGF regulates Xbra expression during Xenopus gastrulation. EMBO J. 13, 4469-4481 (1994).

21. Onichtchouk, D. et al. Silencing of TGF- $\beta$ signalling by the pseudoreceptor BAMBI. Nature 401, 480-485 (1999).

22. Tsang, M., Friesel, R., Kudoh, T. \& Dawid, I. B. Identification of Sef, a novel modulator of FGF signaling. Nature Cell Biol. 4, 165-169 (2002).

23. Fürthauser, M., Lin, W., Ang, S. L., Thisse, B. \& Thisse, C. Sef is a feedback-induced antagonist of Ras/ MAPK-mediated FGF signalling. Nature Cell Biol. 4, 170-174 (2002).

24. Dowd, C. J., Cooney, C. L. \& Nugent, M. A. Heparan sulfate mediates bFGF transport through basement membrane by diffusion with rapid reversible binding. J. Biol. Chem. 8, 5236-5244 (1999).

25. Launay, C., Fromentoux, V., Shi, D. L. \& Boucaut, J. C. A truncated FGF receptor blocks neural induction by endogenous Xenopus inducers. Development 122, 869-880 (1996).

26. Streit, A., Berliner, A. J., Papanayotou, C., Sirulnik, A. \& Stern, C. D. Initiation of neural induction by FGF signalling before gastrulation. Nature 406, 74-78 (2000).

27. Tazaki, A. et al. Neural network in planarian revealed by an antibody against planarian synaptotagmin homologue. Biochem. Biophys. Res. Commun. 260, 426-432 (1999).

28. Sakai, F., Agata, K., Orii, H. \& Watanabe, K. Organization and regeneration ability of spontaneous supernumerary eyes in planarians-Eye regeneration field and pathway selection by optic nerves. Zool Sci. 17, 375-381 (2000).

29. Taira, M., Otani, H., Saint-Jeannet, J. P. \& Dawid, I. B. Role of the LIM class homeodomain protein Xlim-1 in neural and muscle induction by the Spemann organizer in Xenopus. Nature 372, 677-679 (1994).

30. Harland, R. M. in Methods in Cell Biology (eds Kay, B. K. \& Peng, H. B.) 36, 685-695 (Academic, San Diego, 1991).

Supplementary Information accompanies the paper on Nature's website

( http://www.nature.com/nature)

Acknowledgements We thank S. Kuratani for comments on the manuscript, J. Brockes and T. Miyata for promoting collaboration, and D. Turner for pCS $2+$ and pCS $2+n \beta$-gal. This work was supported by Special Coordination Funds for Promoting Science and Technology (K.A.), a Grant-in-Aid for Creative Basic Research (K.A. and T.G.), a Grant-in-Aid for Scientific Research on Priority Areas (K.A. and M.T.) and the National Institutes of Health National Institute of General Medical Sciences (A.S.A.).

Competing interests statement The authors declare that they have no competing financial interests.

Correspondence and requests for materials should be addressed to K.A.

(e-mail: agata@cdb.riken.go.jp). The Dugesia ndk cDNA sequence has been deposited in DDBJ under accession number AB071948.

\section{The polycomb group protein EZH2 is involved in progression of prostate cancer}

\author{
Sooryanarayana Varambally ${ }^{\star} \dagger$, Saravana M. Dhanasekaran ${ }^{\star} \dagger$, \\ Ming Zhou*, Terrence R. Barrette*, Chandan Kumar-Sinha*, \\ Martin G. Sanda $\neq \$$, Debashis Ghosh\|, Kenneth J. Pienta $\neq \$ \uparrow$, \\ Richard G. A. B. Sewalt $\#$, Arie P. Otte $\#$, Mark A. Rubin ${ }^{\star} \ddagger \S$ \\ \& Arul M. Chinnaiyan ${ }^{\star} \ddagger \$
}

* Departments of Pathology, $\$$ Urology, || Biostatistics and I Internal Medicine, and $\$$ Comprehensive Cancer Center, University of Michigan Medical School, Ann Arbor, Michigan 48109, USA

\# Swammerdam Institute for Life Sciences, BioCentrum Amsterdam, University of Amsterdam, 1018 TV Amsterdam, The Netherlands

$\dagger$ These authors contributed equally to this work

Prostate cancer is a leading cause of cancer-related death in males and is second only to lung cancer. Although effective surgical and radiation treatments exist for clinically localized prostate cancer, metastatic prostate cancer remains essentially incurable. Here we show, through gene expression profiling ${ }^{1}$, that the polycomb group protein enhancer of zeste homolog $2(\mathrm{EZH} 2)^{2,3}$ is over-

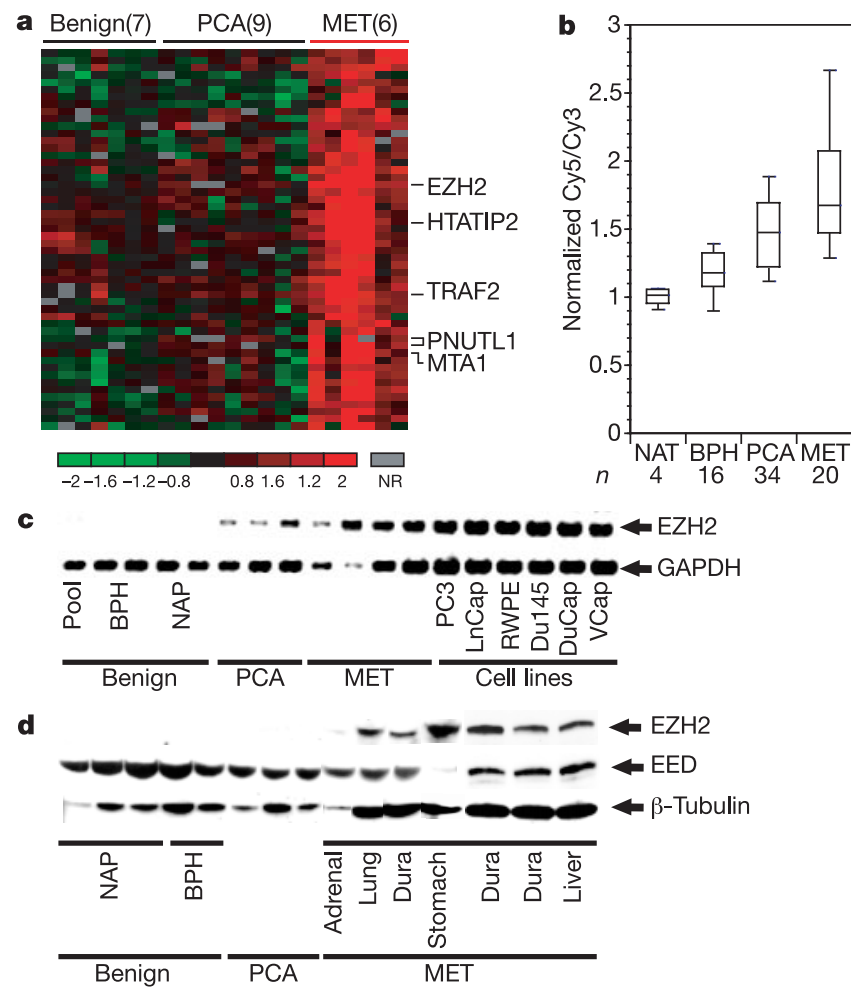

Figure 1 Overexpression of EZH2 in metastatic hormone-refractory prostate cancer (MET). a, Cluster diagram depicting genes that distinguish MET from clinically localized prostate cancer (PCA). Genes upregulated in METs relative to prostate cancer are shown. Red and green represent upregulatiom and downregulation, respectively, relative to the median of the reference pool. Grey represents technically inadequate or missing data, and black represents equal expression relative to the reference sample. $\mathbf{b}$, DNA microarray analysis of prostate cancer shows upregulation of EZH2 in METs. BPH, benign prostatic hyperplasia; NAT, normal adjacent prostate tissue. c, RT-PCR analysis of the EZH2 transcript in prostate tissue and cell lines. $\mathbf{d}$, Increased expression of EZH2 protein in METs. Immunoblot analysis of EZH2 and EED in prostate tissue extracts. The site of metastasis is indicated. 
expressed in hormone-refractory, metastatic prostate cancer. Small interfering RNA (siRNA) duplexes ${ }^{4}$ targeted against $\mathrm{EZH} 2$ reduce the amounts of $\mathrm{EZH} 2$ protein present in prostate cells and also inhibit cell proliferation in vitro. Ectopic expression of $\mathrm{EZH} 2$ in prostate cells induces transcriptional repression of a specific cohort of genes. Gene silencing mediated by $\mathrm{EZH} 2$ requires the SET domain and is attenuated by inhibiting histone deacetylase activity. Amounts of both EZH2 messenger RNA and EZH2 protein are increased in metastatic prostate cancer; in addition, clinically localized prostate cancers that express higher concentrations of $\mathrm{EZH} 2$ show a poorer prognosis. Thus, dysregulated expression of EZH2 may be involved in the progression of prostate cancer, as well as being a marker that distinguishes indolent prostate cancer from those at risk of lethal progression.

Perturbation of the transcriptional 'memory' of a cell can lead to severe developmental defects ${ }^{5,6}$. Lack of differentiation, or anaplasia, is a hallmark of cancer that results from normal cells 'forgetting' their cellular identity. Thus, it is not surprising that dysregulation of the transcriptional maintenance system can lead to malignancy ${ }^{6-8}$. Two groups of proteins have been implicated in maintaining homeotic gene expression and include polycomb (PcG) and trithorax (trxG) group proteins ${ }^{9,10}$. PcG proteins act in large complexes and are thought to repress gene expression, whereas trxG proteins are operationally defined as antagonists of $\mathrm{PcG}$ proteins and thus activate gene expression ${ }^{6,9}$. In human malignancies, $\mathrm{PcG}$ and trxG proteins have been found to be dysregulated mainly in cells of haematopoietic origin ${ }^{11-13}$. EZH2 is the human homologue of the
Drosophila protein Enhancer of Zeste $(\mathrm{E}(\mathrm{z}))^{2}$, which genetic data define as a PcG protein with additional trxG properties ${ }^{10}$. $\mathrm{E}(\mathrm{z})$ and EZH2 contain a SET domain, a highly conserved domain found in chromatin-associated regulators of gene expression that often modulate cell growth pathways ${ }^{14}$.

We previously reported gene expression profiles of benign prostate, clinically localized prostate cancer and metastatic prostate cancer ${ }^{1}$. To characterize genes that specifically mark the molecular transition from organ-confined prostate cancer to metastatic prostate cancer, we implemented a statistical technique called significance analysis of microarrays $(\mathrm{SAM})^{15}$. This approach identified 55 genes that were significantly upregulated (Fig. 1a) and 480 genes that were significantly downregulated (Supplementary Information) in metastatic prostate cancer relative to localized prostate cancer (at a false discovery rate (FDR) of $0.9 \%$ on microarrays containing 10,000 genes). Notably, the gene at the top of the 'list' of upregulated genes in metastatic prostate cancer was EZH2, with a $d$ score $^{15}$ of 4.58 and a gene-specific FDR of 0.0012 (see Supplementary Information for the complete SAM analysis). Fig. 1b summarizes the gene expression of EZH2 in 74 prostate tissue specimens analysed on DNA microarrays ${ }^{1}$. The EZH2 transcript was significantly increased in metastatic prostate cancer with respect to clinically localized prostate cancer (Mann-Whitney test, $P=0.001)$ and benign prostate $(P=0.0001)$.

To validate the DNA microarray results, we carried out polymerase chain reaction with reverse transcription (RT-PCR) on 18 prostate samples and cell lines. As expected, the levels of EZH2 mRNA were increased in malignant relative to benign prostate a

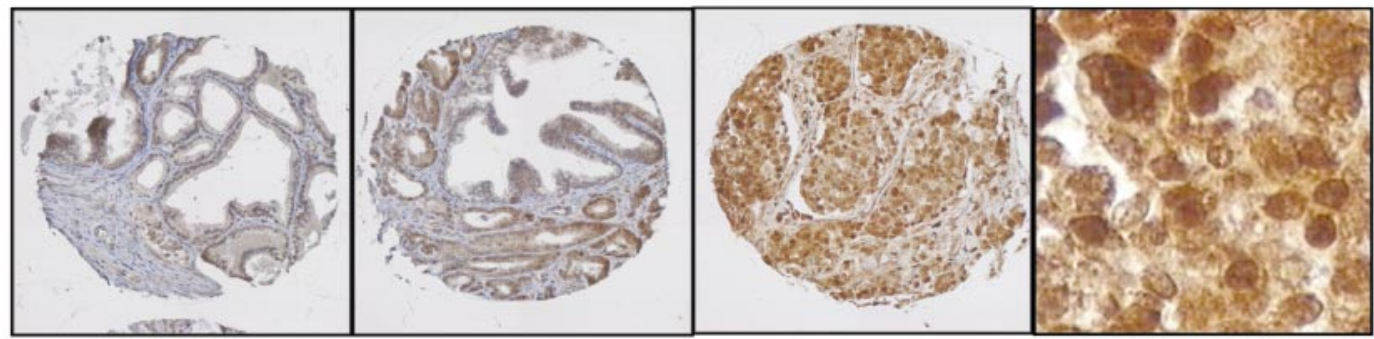

b

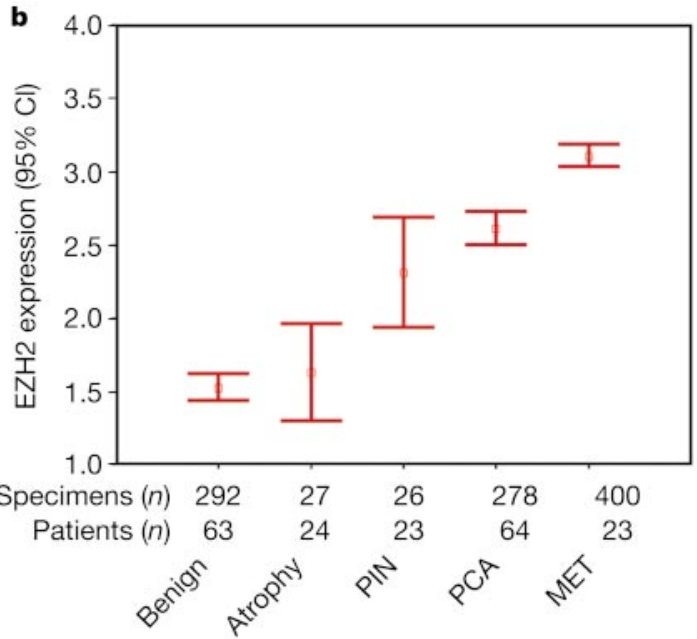

Figure 2 Amounts of EZH2 protein correlate with the aggressiveness of prostate cancer. a, Representative tissue microarray elements stained with antibody to EZH2. Immunohistochemical stains show weak or absent nuclear staining of benign prostate cancer (left, original magnification $\times 100$ ), and strong nuclear staining in metastatic hormone-refractory prostrate cancers (centre right and right, original magnification $\times 100$ and $\times 1,000$, respectively). Also shown is a benign prostate adjacent to prostate

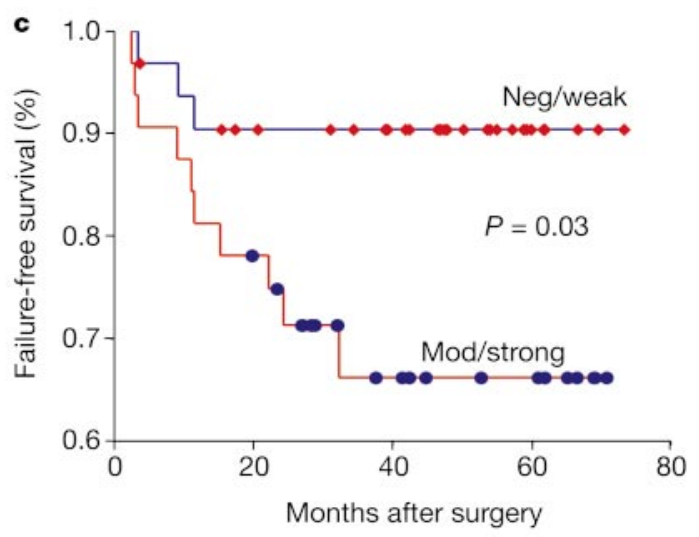

cancer (centre left, original magnification $\times 100$ ). $\mathbf{b}$, Tissue microarray analysis of EZH2 expression. The mean EZH2 protein expression for the indicated prostate tissues is summarized using error bars with 95\% confidence intervals. c, Kaplan-Meier analysis shows that individuals with clinically localized prostate cancer that have high expression of EZH2 (moderate to strong staining) have a greater risk for recurrence of prostate cancer after prostatectomy (log rank test, $P=0.03$ ). 
samples (Fig. 1c). We also analysed tissue extracts by immunoblotting. The amounts of EZH2 protein were markedly increased in metastatic prostate cancer relative to localized prostate cancer or benign prostate (Fig. 1d). Notably, EED, a PcG protein that forms a complex with EZH2 (refs 16, 17), did not show similar protein dysregulation.

We evaluated the expression of EZH2 protein in a wide range of prostate tissues ( $n=1,023$ tissue microarray elements) to determine the extent of its expression in situ (Fig. 2a, b). Of these samples, 400 were from 23 individuals who died of hormonerefractory metastatic prostate cancer ${ }^{18}$. When highly expressed, EZH2 was observed mainly in the nucleus (Fig. 2a, right), as suggested previously ${ }^{11}$. The intensity of EZH2 staining increased from benign, prostatic atrophy, prostatic intraepithelial neoplasia, to clinically localized prostate cancer, with a respective median staining intensity of 1.5 (standard error (s.e.) $0.04,95 \%$ confidence interval (CI) 1.4-1.6), 1.6 (s.e. $0.2,95 \%$ CI 1.3-2.0), 2.3 (s.e. 0.2, 95\%CI 1.9-2.7) and 2.6 (s.e. 0.1, 95\%CI, 2.5-2.7), respectively (Fig. $2 \mathrm{~b})$. The strongest EZH2 expression was in metastatic prostate cancer, with a median staining intensity of 3.1 (s.e. $0.03,95 \%$ CI 3.0 3.2). There was a statistically significant difference in EZH2 staining intensity between benign prostate tissue and localized prostate cancer (analysis of variance (ANOVA) post-hoc analysis mean difference 1.0, $P<0.0001)$. Likewise, metastatic prostate cancer had significantly higher expression of EZH2 than did clinically localized prostate cancer (ANOVA post-hoc analysis mean difference $0.5, P<0.0001)$. These findings suggested that as prostate neoplasia progresses there is a trend towards increased expression of EZH2 protein. They also suggested that EZH2 concentrations might indicate how aggressive an individual's prostate cancer is, given that the highest expression was observed in hormone-refractory, metastatic prostate cancer.

We therefore examined whether EZH2 protein could be used to predict clinical outcome in men treated with surgery for clinically localized prostate cancer. Using a previously validated tissue microarray ${ }^{19}$, we examined 278 specimens from 64 individuals with clinical follow-up. To test the utility of EZH2 as a potential tissue biomarker for prostate cancer, we examined the clinical outcome of these 64 cases, taking into account clinical and pathological parameters. Clinical failure was defined as either an increase of $0.2 \mathrm{ng} \mathrm{ml}^{-1}$ prostate-specific antigen (PSA) or recurrence of disease after prostatectomy (for example, development of metastatic disease).

By Kaplan-Meier analysis (Fig. 2c), an EZH2 staining intensity of 3 or higher was associated significantly with clinical failure in $31 \%$ (10/32) of individuals ( $\log \operatorname{rank} P=0.03$ ), whereas a staining intensity of less than 3 was found in $9 \%(3 / 32)$ of individuals with clinical failure. There was no significant correlation between EZH2 amounts and Gleason score, tumour stage or surgical margin status. Notably, there was a significant $(P=0.048)$ albeit weak (Pearson coefficient $=0.33$ ) correlation between EZH2 protein and proliferation index in situ, as assessed by the Ki-67 labelling index (Supplementary Information). Multivariable Cox hazards regression analysis showed that EZH2 protein expression $(\geq 3$ versus $<3$ ) was the best predictor of clinical outcome with a recurrence ratio of $4.6(95 \% \mathrm{CI} 1.2-17.1, P=0.02)$, which was significantly better than surgical margin status, maximum tumour dimension, Gleason score and pre-operative PSA (Supplementary Information). Thus, monitoring the amounts of EZH2 protein in prostate specimens may provide additional prognostic information that is not discernible with current clinical and pathology parameters alone.

To examine the role of EZH2 in prostate cancer progression, we used RNA interference (RNAi) to disrupt EZH2 expression in transformed prostate cells in vitro. We designed duplexes of 21nucleotide RNA (siRNAs) to target EZH2 (ref. 4) and tested them on the transformed androgen-responsive prostate cell line $\mathrm{RWPE}^{20,21}$ and the metastatic prostate cancer cell line PC3. After a

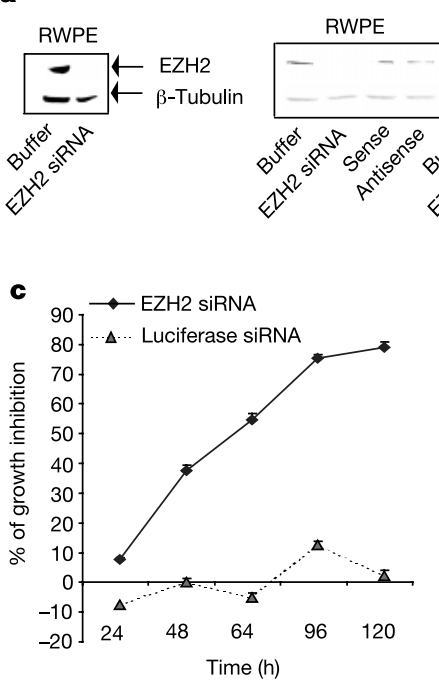

PC3

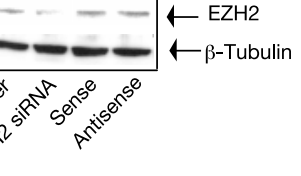

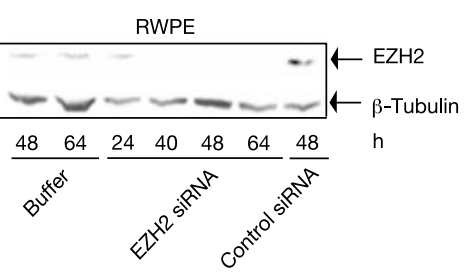

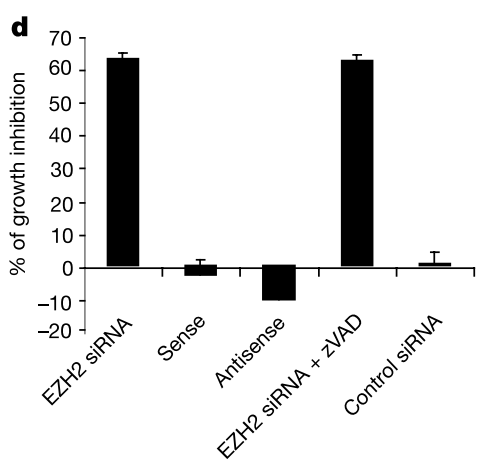

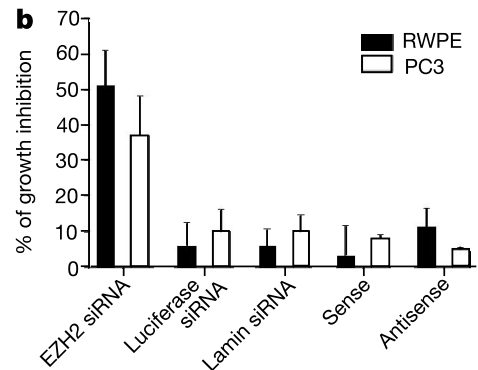

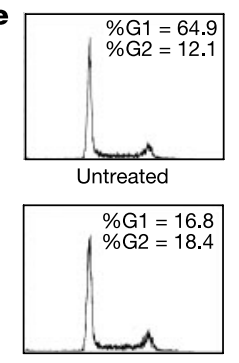

Antisense

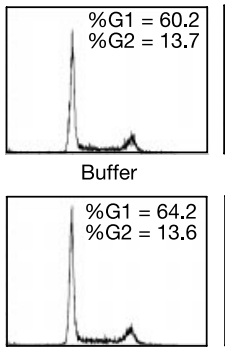

Luciferase siRNA

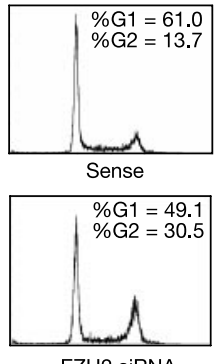

EZH2 siRNA
Figure $3 \mathrm{~A}$ role for $\mathrm{EZH} 2$ in prostate cell proliferation. a, Immunoblot analysis of prostate cells (RWPE, PC3) incubated with EZH2 siRNA duplexes. Sense and antisense oligonucleotides of EZH2 and unrelated siRNA duplexes were used as controls. $\mathbf{b}$, RNAi of EZH2 decreases cell proliferation, as assessed by cell counting assay after $48 \mathrm{~h}$ of incubation with EZH2 siRNA. Significant inhibition of growth is seen in cells treated with EZH2 siRNA relative to those treated with unrelated controls (Student's $t$-test, $P<0.001$ ). Data are from two independent experiments carried out in quadruplicate. c, Time course of growth inhibition by EZH2 siRNA. RWPE cells were treated with EZH2 or luciferase siRNA for indicated time periods and the cells were counted. d, RNAi of EZH2 inhibits cell proliferation, as assessed by WST assay. EZH2 siRNA significantly inhibited cell proliferation as compared with controls (Student's $t$-test, $P<0.001$ ). e, RNAi of EZH2 induces cell-cycle arrest of prostate cells as assessed by flow cytometry. 
$48 \mathrm{~h}$ of transfection with siRNA duplexes, quantification of the amounts of endogenous EZH2 protein showed a specific downregulation of protein in prostate cell lines (Fig. 3a). Sense or antisense oligonucleotides, as well as unrelated siRNA duplexes, did not affect the amounts of EZH2 (Fig. 3a, middle and right), verifying the specificity of the siRNA approach.

We next examined the phenotype of the prostate cells with reduced EZH2 expression. RWPE cell counts taken $48 \mathrm{~h}$ after transfection with siRNA showed a marked (62\%) inhibition of cell growth caused by the EZH2 siRNA duplex, whereas the corresponding sense and antisense EZH2 oligonucleotides or unrelated duplexes showed minimal inhibition (Fig. 3b). The PC3 prostate cancer cell line showed a similar growth inhibition by EZH2 siRNA, indicating that these findings were not specific to the RWPE cell line (Fig. 3b). The effect of EZH2 siRNA on prostate cell growth was monitored at time points ranging from 24 to $120 \mathrm{~h}$ (Fig. 3c), during which over $80 \%$ growth inhibition was observed.

Using a commercially available cell proliferation reagent WST-1, which measures mitochondrial dehydrogenase activity, we observed a decrease in cell proliferation in cells transfected with the EZH2 siRNA duplex but not with the unrelated duplexes (Fig. $3 \mathrm{~d}$ ). In the time frame considered $(48 \mathrm{~h})$, RNAi of EZH2 did not induce apoptosis, as assessed by propidium iodide staining of nuclei or cleavage of poly(adenosine diphosphate)-ribosylpolymerase (data not shown). Consistent with this, the broad-spectrum caspase inhibitor, zVAD-fmk, failed to attenuate the inhibition of cell proliferation induced by EZH2 siRNA (Fig. 3d). Notably, flow cytometric analysis of prostate cells treated with EZH2 siRNA showed cell-cycle arrest in the G2/M phase (Fig. 3e). Unrelated control siRNA duplexes failed to induce a similar dysregulation of the cell cycle. Together, these observations indicate that EZH2 may be involved in the proliferation of prostate cells by mitigating the G2/M transition.

To understand further the functional role of EZH2 in prostate cells, we generated an epitope-tagged version of wild-type EZH2 and a deletion mutant of EZH2 lacking the conserved SET domain ${ }^{14}$ in the eukaryotic expression vector, pcDNA3 (Fig. 4a, b). We also designed an 'inducible' version ${ }^{22,23}$ of EZH2 by creating a fusion protein with a modified murine oestrogen receptor (ER; Fig. 4a, b).

PcG proteins have been proposed to mediate their functions by repressing target genes ${ }^{2,5}$. To test this hypothesis, we transiently transfected RWPE prostate cells with wild-type EZH2 and monitored alterations in global gene expression using DNA microarrays. The RNA from the transfected cell line was labelled with one fluorescent dye, whereas the paired reference sample was labelled with a second distinguishable fluorescent dye. By making direct comparisons between 'gene'-transfected cell lines and cells lines transfected with control vector, we emphasized the molecular differences between the samples and obviated the need for complete transfection efficiency.

When EZH2 was overexpressed in RWPE cells or SUM149 breast carcinoma cells, there was a consistent repression of a cohort of genes (Fig. 4c, d). When compared with vector-transfected cells, the only gene that was upregulated significantly in EZH2-transfected cells was EZH2 itself (Fig. 4c). The EZH2-mediated transcriptional repression was dependent on an intact SET domain (Fig. 4c), because deletion of this domain did not produce a repressive phenotype and in some cases 'de-repressed' genes. EZH2 interacts with histone deacetylase 2 (HDAC2) through the EED protein ${ }^{24}$, and in our system EZH2-mediated gene silencing was dependent on HDAC activity, because the commonly used HDAC inhibitor trichostatin A (TSA) completely abrogated the effects of EZH2 (Fig. 4c). Thus, EZH2 function requires both an intact SET domain and endogenous HDAC activity.

To identify genes that are significantly repressed by EZH2, we compared cells transfected with wild-type EZH2 with cells trans-
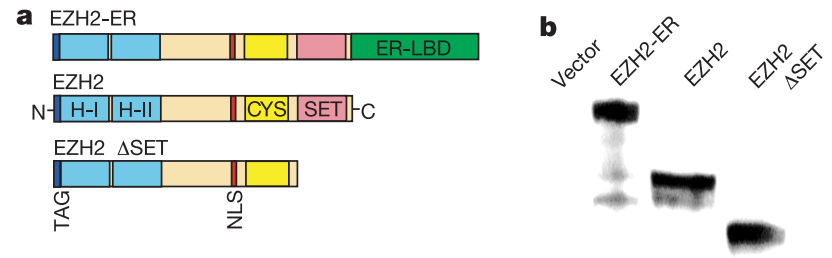

c
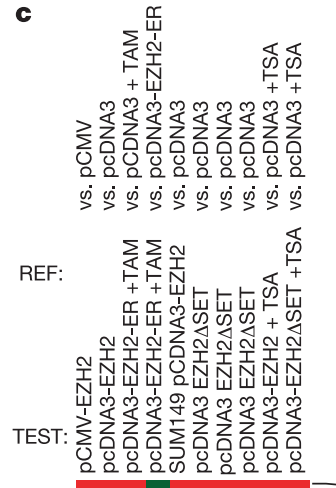

d Significant: 161

False significant: 0.739

delta:0.452

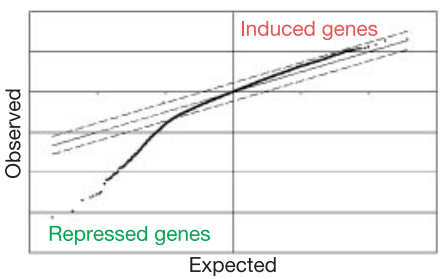

Expected

EZH2 2 alkaline phosphatase

WASF1 WAS protein family

AMACR alpha-methylacyl-CoA racemase

RAC3 ras-related C3

VIL2 villin 2 ezrin

CA6 carbonic anhydrase VI

GSTM4 glutathione S-transferase M4

RNF5 ring finger protein 5

- SCAMP2 secretory carrier membrane protein 2

- CLDN10 claudin 10

ANXA6 annexin A6

LOC51714 selenoprotein T

KIAA0105 Wilms' tumour 1-associating protein

ZNFN1A1 zinc finger protein subfamily $1 \mathrm{~A}$

CDC27 cell division cycle 27

FRZB frizzled-related protein

- SPIB Spi-B transcription factor

- PRG2 proteoglycan 2

CA5A carbonic anhydrase $V$

ZNF262 zinc finger protein 262

TNA tetranectin plasminogen-binding protein

FBLN1 fibulin 1

RAB6A member RAS oncogene family

OXTR oxytocin receptor

KRT6B keratin 6B

SFRP4 secreted frizzled-related protein 4

CSTF2 cleavage stimulation factor, subunit 2

FOXF2 forkhead box F2

CPD carboxypeptidase D

SCP2 sterol carrier protein 2

EPC enhancer of polycomb

NME5 non-metastatic cells 5

ITGA1 integrin, alpha 1

MNDA myeloid cell nuclear differentiation ag.

CSF3R colony stimulating factor 3 receptor

PCDH20 protocalinering

CSF3R colony stimulating factor 3 receptor

CDC27 cell division cycle 27

IL10RA interleukin 10 receptor, alpha

ICAM1 CD54 intercellular adhesion molecule 1

APPBP2 amyloid beta cytoplasmic tail-BP 2

RBM5 RNA binding motif protein 5

RNF15 ring finger protein 15

EST, Homo sapiens $14 q 32$ Jagged2 gene

SGPL1 sphingosine-1-phosphate lyase 1

SGPL1 sphingosine-1-phosp
ZNF42 zinc finger protein 42

ITM1 integral membrane protein 1

ERBB2 v-erb-b2

PPP1R12C protein phosphatase 1

MMP7 matrix metalloproteinase 7

HSD11B2 hydroxysteroid 11-beta dehydrogenase 2

DDT D-dopachrome tautomerase

ARHGAP1 Rho GTPase activating protein 1

Figure 4 EZH2 functions as a transcriptional repressor in prostate cells. a, EZH2 constructs. CYS, cysteine-rich domain; ER, ligand-binding domain of the oestrogen receptor; $\mathrm{H}-\mathrm{I}$ and $\mathrm{H}-\mathrm{II}$, homology domains that share similarity with $\mathrm{E}(\mathrm{z}) ; \mathrm{NLS}$, nuclear localization signal; SET, SET domain; TAG, Myc epitope tag. b. Expression of the EZH2 constructs. c, Cluster diagram of genes significantly repressed by overexpression of EZH2. See Fig. 1a for the matrix colour scheme and Supplementary Information for the complete data set. TAM, tamoxifen; TSA, trichostatin A. d, SAM analysis ${ }^{15}$ of gene expression profiles of cells transfected with EZH2 versus cells transfected with EZH2 $\triangle \mathrm{SET}$ 
fected with EZH2 $\Delta$ SET. We found that 163 genes were consistently repressed, whereas no genes were activated at an FDR of 0.0045 (Fig. 4d, and Supplementary Information). The list of genes that were repressed indicates several intriguing associations, including EZH2-mediated repression of specific PcG proteins, transcription factors and cell-cycle regulators (Supplementary Information).

In summary, we have identified the association of EZH2 with advanced prostate cancer by gene expression profiling of tumour specimens from individuals who died of metastatic disease. Measuring EZH2 amounts (in prostate cancer specimens) might have potential as a molecular determinant of prostate cancer progression and metastasis. In addition, we have shown that EZH2 has a role in mediating cell proliferation and transcriptional repression in prostate cells. Overall, our study indicates that dysregulation of the transcriptional memory machinery may contribute to the lethal progression of prostate cancer and may provide a potential mechanism for the constellation of genes repressed in metastatic disease. $\square$

\section{Methods}

\section{SAM analysis of prostate cancer gene expression}

We carried out SAM analysis by comparing gene expression profiles of metastatic prostate cancer samples with those of clinically localized prostate cancer samples from our previous work $^{1}$. Genes were analysed using Cluster ${ }^{25}$, implementing average linkage hierarchical clustering of genes, and the output (Supplementary Information) was visualized by Treeview ${ }^{25}$.

\section{RT-PCR}

We carried out RT-PCR amplifications with $1 \mu \mathrm{g}$ of total RNA isolated from the indicated prostate tissues and cell lines. Primer sequences are given in the Supplementary Information.

\section{Immunoblot analysis}

Prostate tissue extracts were separated by SDS-PAGE and blotted onto nitrocellulose membranes. Antibodies against EZH2 and EED (ref. 17) and a polyclonal antibody against $\beta$-tubulin (Santa Cruz) were used at 1:1,000 dilution for immunoblot analysis.

\section{Tissue microarray analysis}

The clinically stratified prostate cancer tissue microarrays used in this study have been described ${ }^{1,19,26}$. Tissues were from the radical prostatectomy series at the University of Michigan and from the Rapid Autopsy Program, which are both part of Michigan Prostate SPORE Tissue Core. We obtained Institutional Review Board approval to procure and analyse the tissues used in this study.

We evaluated EZH2 protein expression on a wide range of prostate tissue to determine the intensity and extent of expression in situ. Immunohistochemistry was carried out on three high-density tissue microarrays containing samples of benign prostate, prostatic atrophy, prostatic intraepithelial neoplasia, clinically localized prostate cancer and metastatic prostate cancer. We used standard biotin-avidin complex

immunohistochemistry to evaluate EZH2 protein expression using an antibody against EZH2. Protein expression was scored as negative (score $=1)$, weak $(2)$, moderate $(3)$ and strong (4). Four replicate tissue cores were sampled from each of the selected tissue types. EZH2 expression was evaluated in a blind manner separately by M.A.R. and M.Z. using a validated web-based tool ${ }^{26,27}$, and the median value of all measurements from a single individual was used for subsequent analysis. Staining assessment was highly reproducible between the two pathologists with a $\kappa$ value of 0.73 .

\section{Clinical outcomes analysis}

To assess individual variables for risk of recurrence, we used Kaplan-Meier survival analysis and created a univariate Cox proportional hazards model. PSA recurrence was defined as greater than $0.2 \mathrm{ng} \mathrm{ml}^{-1}$ after radical prostatectomy. Covariates included Gleason sum, preoperative PSA, maximum tumour dimension, tumour stage and surgical margin status. To assess the influence of several variables simultaneously, including EZH2 protein expression, we developed a final multivariate Cox proportional hazards model of statistically significant covariates. Statistical significance in univariate and multivariate Cox models was determined by Wald's test. A $P$ value of less than 0.05 was considered statistically significant.

\section{EZH2 constructs}

Myc-tagged EZH2-pCMV was a gift from T. Jenuwein. The Myc-EZH2 fragment was subcloned into the expression vector pCDNA3 (Invitrogen). The EZH2-ER and $\mathrm{EZH} 2 \Delta \mathrm{SET}$ constructs are described in the Supplementary Information.

\section{RNAi}

We chemically synthesized 21-nucleotide sense and antisense RNA oligonucleotides (Dharmacon) and annealed them to form duplexes. The EZH2 siRNA was targeted to the region corresponding to residues 85-106 of human EZH2 (NM004456). Control siRNA duplexes targeted luciferase, lamin and AMACR (NM014324). The human transformed prostate cell lines RWPE ${ }^{20}$ and PC3 were plated at $2 \times 10^{5}$ cells per well in a 12 -well plate for immunoblot analysis, cell counts and FACS analysis, and at $1.5 \times 10^{4}$ cell per well in a 96-well plate for WST-1 proliferation assays. Twelve hours after plating, the cells were transfected with $60 \mathrm{pmol}$ of siRNA duplex, sense or antisense oligonucleotides using oligofectamine (Invitrogen). We carried out a second identical transfection $24 \mathrm{~h}$ later. At certain time points after the first transfection, the cells were lysed for immunoblot analysis and treated with trypsin for estimating cell numbers or FACS analysis. For cell counts at 96 and $120 \mathrm{~h}$, the cells were treated with trypsin and replated in 6-well dishes $64 \mathrm{~h}$ after the first transfection.

\section{Cell proliferation assays}

Cell proliferation was determined by a colorimetric assay of cell viability that is based on the cleavage of the tetrazolium salt WST-1 by mitochondrial dehydrogenases (Roche). The absorbance of the formazan dye formed, which correlates with the number of metabolically active cells in the culture, was measured at $450 \mathrm{~nm} 1 \mathrm{~h}$ after adding the reagent. Cell counts were estimated by treating the cells with trypsin, followed by analysis on a Coulter cell counter at specified time points.

\section{Flow cytometric analysis}

Trypsin-treated cells were washed with PBS and fixed in 70\% ethanol overnight for FACS analysis. Before staining with propidium iodide, the cells were washed again with PBS and analysed by flow cytometry.

\section{Microarray analysis of EZH2-transfected cells}

Initial testing of the transient transfection analysis system (data not shown) showed that overexpression of TNFR1 (p55) induced similar expression profiles to those observed after incubating cells with $\mathrm{TNF}^{28}$. Samples expressing the EZH $2 \Delta$ SET mutant were compared with those expressing EZH2 using the SAM analysis package (ref. 15 and Supplementary Information).

Received 13 May; accepted 31 July 2002; doi:10.1038/nature01075.

1. Dhanasekaran, S. M. et al. Delineation of prognostic biomarkers in prostate cancer. Nature 412, 822-826 (2001).

2. Laible, G. et al. Mammalian homologues of the Polycomb-group gene Enhancer of zeste mediate gene silencing in Drosophila heterochromatin and at S. cerevisiae telomeres. EMBO J. 16, 3219-3232 (1997).

3. Satijn, D. P. \& Otte, A. P. Polycomb group protein complexes: do different complexes regulate distinct target genes? Biochim. Biophys. Acta 1447, 1-16 (1999).

4. Elbashir, S. M. et al. Duplexes of 21-nucleotide RNAs mediate RNA interference in cultured mammalian cells. Nature 411, 494-498 (2001).

5. Jacobs, J. J. \& van Lohuizen, M. Cellular memory of transcriptional states by Polycomb-group proteins. Semin. Cell. Dev. Biol. 10, 227-235 (1999).

6. Francis, N. J. \& Kingston, R. E. Mechanisms of transcriptional memory. Nature Rev. Mol. Cell Biol. 2, 409-421 (2001).

7. Jacobs, J. J., Kieboom, K., Marino, S., DePinho, R. A. \& van Lohuizen, M. The oncogene and Polycomb-group gene $b m i-1$ regulates cell proliferation and senescence through the ink $4 a$ locus. Nature 397, 164-168 (1999).

8. Jacobs, J. J. et al. Bmi-1 collaborates with c-Myc in tumorigenesis by inhibiting c-Myc-induced apoptosis via INK4a/ARF. Genes Dev. 13, 2678-2690 (1999).

9. Mahmoudi, T. \& Verrijzer, C. P. Chromatin silencing and activation by Polycomb and trithorax group proteins. Oncogene 20, 3055-3066 (2001).

10. LaJeunesse, D. \& Shearn, A. E(z): a polycomb group gene or a trithorax group gene? Development 122, 2189-2197 (1996).

11. Raaphorst, F. M. et al. Coexpression of BMI-1 and EZH2 polycomb group genes in Reed-Sternberg cells of Hodgkin's disease. Am. J. Pathol. 157, 709-715 (2000).

12. van Lohuizen, M. et al. Identification of cooperating oncogenes in $\mathrm{E} \mu$-myc transgenic mice by provirus tagging. Cell 65, 737-752 (1991).

13. Haupt, Y., Alexander, W. S., Barri, G., Klinken, S. P. \& Adams, J. M. Novel zinc finger gene implicated as myc collaborator by retrovirally accelerated lymphomagenesis in $\mathrm{E} \mu$-myc transgenic mice. Cell 65, 753-763 (1991).

14. Jenuwein, T., Laible, G., Dorn, R. \& Reuter, G. SET domain proteins modulate chromatin domains in eu- and heterochromatin. Cell. Mol. Life Sci. 54, 80-93 (1998).

15. Tusher, V. G., Tibshirani, R. \& Chu, G. Significance analysis of microarrays applied to the ionizing radiation response. Proc. Natl Acad. Sci. USA 98, 5116-5121 (2001).

16. van Lohuizen, M. et al. Interaction of mouse polycomb-group (Pc-G) proteins Enx1 and Enx2 with Eed: indication for separate Pc-G complexes. Mol. Cell. Biol. 18, 3572-3579 (1998).

17. Sewalt, R. G. et al. Characterization of interactions between the mammalian polycomb-group proteins Enx1/EZH2 and EED suggests the existence of different mammalian polycomb-group protein complexes. Mol. Cell. Biol. 18, 3586-3595 (1998).

18. Rubin, M. A. et al. Rapid ('warm') autopsy study for procurement of metastatic prostate cancer. Clin. Cancer Res. 6, 1038-1045 (2000).

19. Perrone, E. E. et al. Tissue microarray assessment of prostate cancer tumour proliferation in AfricanAmerican and white men. J. Natl Cancer Inst. 92, 937-939 (2000).

20. Webber, M. M., Bello, D., Kleinman, H. K. \& Hoffman, M. P. Acinar differentiation by non-malignant immortalized human prostatic epithelial cells and its loss by malignant cells. Carcinogenesis 18, 1225-1231 (1997).

21. Bello, D., Webber, M. M., Kleinman, H. K., Wartinger, D. D. \& Rhim, J. S. Androgen responsive adult human prostatic epithelial cell lines immortalized by human papillomavirus 18. Carcinogenesis 18, 1215-1223 (1997).

22. Littlewood, T. D., Hancock, D. C., Danielian, P. S., Parker, M. G. \& Evan, G. I. A modified oestrogen receptor ligand-binding domain as an improved switch for the regulation of heterologous proteins. Nucleic Acids Res. 23, 1686-1690 (1995).

23. Juin, P., Hueber, A. O., Littlewood, T. \& Evan, G. c-Myc induced sensitization to apoptosis is mediated through cytochrome c release. Genes Dev. 13, 1367-1381 (1999). 
24. van der Vlag, J. \& Otte, A. P. Transcriptional repression mediated by the human polycomb-group protein EED involves histone deacetylation. Nature Genet. 23, 474-478 (1999).

25. Eisen, M. B., Spellman, P. T., Brown, P. O. \& Botstein, D. Cluster analysis and display of genome-wide expression patterns. Proc. Natl Acad. Sci. USA 95, 14863-14868 (1998).

26. Manley, S., Mucci, N. R., De Marzo, A. M. \& Rubin, M. A. Relational database structure to manage high-density tissue microarray data and images for pathology studies focusing on clinical outcome: the prostate specialized program of research excellence model. Am. J. Pathol. 159, 837-843 (2001).

27. Bova, G. S. et al. Web-based tissue microarray image data analysis: initial validation testing through prostate cancer Gleason grading. Hum. Pathol. 32, 417-427 (2001).

28. Kumar-Sinha, C., Varambally, S., Sreekumar, A. \& Chinnaiyan, A. M. Molecular cross-talk between the TRAIL and interferon signaling pathways. J. Biol. Chem. 277, 575-585 (2001).

Supplementary Information accompanies the paper on Nature's website ( http://www.nature.com/nature).

Acknowledgements This study was made possible by tissues donated by patients with metastatic prostate cancer enrolled in the University of Michigan, Rapid Autopsy Program funded by the Specialized Program of Research Excellence (SPORE) in Prostate Cancer at the National Cancer Institute. We thank J. Wei for clinical data collection; K. Hamer for preparing polycomb

antibodies; R. Kunkel for figure preparation; J. Harwood and M. LeBlanc for technical assistance; A. Menon for sequence verification; and C. Ingold and G. Tueckmantel for database assistance. A.M.C. is a Pew Foundation Scholar. This work is supported in part by grants from the NIH (A.M.C.), CaPCURE (A.M.C.) and the Michigan SPORE in Prostate Cancer (K.P., M.A.R., A.M.C. and M.G.S.).

Competing interests statement The authors declare that they have no competing financial interests.

Correspondence and requests for materials should be addressed to A.M.C. (e-mail: arul@umich.edu).

\section{Class IV semaphorin Sema4A enhances T-cell activation and interacts with Tim-2}

\author{
Atsushi Kumanogoh ${ }^{\star}$, Satoko Marukawa ${ }^{\star} \dagger \neq$, Kazuhiro Suzuki ${ }^{\star} \dagger$, \\ Noriko Takegahara*, Chie Watanabe ${ }^{\star}$, EweSeng Ch'ng ${ }^{\star}$, Isao Ishida*, \\ Harutoshi Fujimura $\$$, Saburo Sakoda $\$$, Kanji Yoshida* \& \\ Hitoshi Kikutani* \\ * Department of Molecular Immunology, Research Institute for Microbial \\ Diseases, Osaka University, 3-1 Yamada-oka, Suita, Osaka 565-0871, Japan \\ $\ddagger$ Department of Internal Medicine and Molecular Science, and \\ $\$$ Department of Neurology, Graduate School of Medicine, Osaka University, \\ 2-2 Yamada-oka, Suita, Osaka 565-0871, Japan \\ $\dagger$ These authors contributed equally to this work
}

Semaphorins are a family of phylogenetically conserved soluble and transmembrane proteins ${ }^{1,2}$. Although many soluble semaphorins deliver guidance cues to migrating axons during neuronal development ${ }^{3-5}$, some members are involved in immune responses $^{6-9}$. For example, CD100 (also known as Sema4D), a class IV transmembrane semaphorin, signals through CD72 to effect nonredundant roles in immune responses ${ }^{7,10-13}$ in a ligandreceptor system that is distinct from any seen previously in the nervous system ${ }^{14,15}$. Here we report that the class IV semaphorin Sema4A, which is expressed in dendritic cells and B cells, enhances the in vitro activation and differentiation of $\mathrm{T}$ cells and the in vivo generation of antigen-specific $\mathrm{T}$ cells. Treating mice with monoclonal antibodies against Sema4A blocks the development of an experimental autoimmune encephalomyelitis that is induced by an antigenic peptide derived from myelin oligodendrocyte glycoprotein. In addition, expression cloning shows that the Sema4A receptor is Tim-2, a member of the family of T-cell immunoglobulin domain and mucin domain (Tim) proteins that is expressed on activated T cells.

To study the semaphorins expressed in dendritic cells (DCs), we isolated a complementary DNA fragment of the class IV semaphorin, Sema4A, through cloning based on polymerase chain reaction (PCR) using degenerate oligonucleotide primers derived from motifs that are conserved among members of the semaphorin family. Sema4A was originally identified as semB, the expression of which gradually increases during embryonic development ${ }^{16}$, although its functions have not yet been reported. Analysis by PCR with reverse transcription (RT-PCR) showed that there was prominent expression of Sema4A messenger RNA in the brain, spleen, lung, kidney and testis of adult tissues (data not shown).

To investigate the biological function and expression of Sema4A in the immune system, we prepared recombinant soluble mouse Sema4A protein comprising the putative extracellular region fused to the human immunoglobulin- $\gamma 1$ (IgG1) Fc fragment (Sema4A-Fc). We generated antibodies against Sema4A by immunizing rats with Sema $4 \mathrm{~A}-\mathrm{Fc}$ and screening the hybridomas for reactivity to mouse Chinese hamster ovary ( $\mathrm{CHO}$ ) cell transfectants expressing Sema4A (Fig. 1a). The anti-Sema4A antibody (hereafter referred to as anti-Sema4A) specifically bound to Sema4A but not to control $\mathrm{CHO}$ cells carrying only the Neomycin resistance plasmid or to $\mathrm{CHO}$ cells expressing CD100. As expected from our cloning methodology, Sema4A was expressed abundantly on the surface of bone-marrow-derived and splenic DCs (Fig. 1b). No differences were found in Sema4A expression between DCs expressing the CD8 $\alpha$ antigen and those not expressing it. Expression of Sema4A was also detected on the surface of resting B cells but not resting $\mathrm{T}$ cells. When $\mathrm{B}$ cells were stimulated with an antibody against CD40 (anti-CD40), the expression of Sema4A was upregulated. The slight expression of Sema4A became detectable when $\mathrm{T}$ cells were stimulated with an antibody against CD3 (anti-CD3).

To test whether Sema4A has an effect on T-cell activation, $\mathrm{T}$ cells carrying the $\mathrm{CD} 4$ antigen $\left(\mathrm{CD} 4^{+} \mathrm{T}\right.$ cells) were stimulated with immobilized anti-CD3 in the presence of Sema4A-Fc or control human IgG1. Sema4A-Fc enhanced the T-cell proliferation and interleukin 2 (IL-2) production induced by anti-CD3 (Fig. 2a). We next examined whether Sema4A promotes the differentiation of naive $\mathrm{T}$ cells into $\mathrm{T}$-helper $1\left(\mathrm{~T}_{\mathrm{H}} 1\right)$-like or $\mathrm{T}_{\mathrm{H}} 2$-like effector populations under their respective culture conditions ${ }^{17}$. Addition of Sema4A-Fc significantly enhanced the induction of cells producing interferon- $\gamma$ (IFN- $\gamma$ ) and cells producing IL-4 (Fig. 2b). We also examined the effect of Sema4A-Fc on mixed lymphocyte reactions (MLRs) between allogeneic $\mathrm{T}$ cells and DCs. Bonemarrow-derived DCs on a C57BL/6 background were used to stimulate BALB/c spleen $\mathrm{CD}^{+} \mathrm{T}$ cells. Sema4A-Fc enhanced MLRs, including the proliferation of $\mathrm{T}$ cells and the production of IL-2 (Fig. 2c). When fully mature DCs that had been treated with anti-CD40 and fixed with paraformaldehyde were used as MLR stimulants, Sema4A-Fc showed an enhancing effect (Fig. 2d), indicating that Sema4A acts directly on T cells. Anti-Sema4A also inhibited MLRs between allogeneic $\mathrm{T}$ cells and DCs (Fig. 2e), confirming the involvement of Sema4A in T-cell activation through interactions between $\mathrm{T}$ cells and DCs. Although we have shown that CD100 enhances the activation of $\mathrm{B}$ cells and $\mathrm{DCs}^{7,18}$, Sema4A-Fc did not possess this activity (Supplementary Information Fig. 1).

To determine whether Sema4A is involved in antigen-specific T-cell responses in vivo, we immunized mice subcutaneously with keyhole limpet haemocyanin (KLH) in complete Freund's adjuvant (CFA) and then treated them intravenously with Sema4A-Fc for $4 \mathrm{~d}$. Five days after immunization, $\mathrm{CD}^{+}{ }^{+} \mathrm{T}$ cells were prepared from the draining lymph nodes and were tested in vitro for antigenspecific responses. There was a significant increase in proliferation 